\title{
CONTRIBUIÇÃO PARA UMA AGENDA DE ATENÇÃO BÁSICA DE SAÚDE EM TEMPOS DE PANDEMIA DE COVID-19
}

\author{
Revisão rápida
}

\author{
Clóvis Ricardo Mobtenegro de Lima ${ }^{1}$ \\ IBICT \\ clovismlima@gmail.com \\ Eliane Azevedo Gomes ${ }^{2}$ \\ Universidade Federal do Rio de Janeiro \\ elianeagomes@gmail.com
}

Maria Cristiane Barbosa ${ }^{3}$

Universidade de São Paulo. mgalvao@usp.br

Kátia de Oliveira Simões ${ }^{4}$ Instituto Nacional de Câncer katia.simoes@gmail.com

\begin{abstract}
Resumo
Esta revisão rápida busca mapear cuidados básicos em nível da atenção primária que podem e devem ser realizados para enfrentar a pandemia de Covid-19. Assim, considera-se como eixos conceituais da revisão a "atenção primária", a "Covid-19" e os "cuidados básicos de saúde". Dos 177 artigos encontrados na PUBMED, LILACS e Scielo, retirando-se os duplicados e aplicando-se os critérios de inclusão e exclusão, 24 artigos são selecionados. A partir da análise dos estudos, construiu-se uma agenda de atenção básica à saúde com treze pontos em complexidade crescente: moradia com saneamento, água encanada e comida, educação em saúde e vigilância epidemiológica; mobilização de agentes comunitários, médicos de família, profissionais de saúde bucal, profissionais de saúde mental, garantia de equipamentos de proteção individual para todos eles; gestão flexível do trabalho, diretrizes para grupos de vulneráveis e de maior risco de dano e letalidade, clínicas móveis e uso da telemedicina.
\end{abstract}

Palavras-chave: Atenção primária de saúde. Sistema Único de Saúde. Pandemia. Covid-19. Gestão de organizações de saúde. Revisão rápida.

\section{CONTRIBUTION TO A PRIMARY HEALTH CARE AGENDA IN TIMES OF COVID-19 PANDEMIC \\ Rapid review}

\begin{abstract}
This rapid review aims to map basic care at the primary care level that can and should be undertaken to address the Covid-19 pandemic. Thus, the conceptual axes of the review are considered to be "primary care", "Covid19" and "medical care". From PUBMED, LILACS and Scielo Saude Publica databases, 177 articles found, removing duplicate ones and applying the inclusion and exclusion criteria, 24 articles are selected. From the analysis of the studies, a primary health care agenda was built with thirteen points in increasing complexity: housing and sanitation, running water and food, health education, and epidemiological surveillance; to mobilize community agents, family doctors, oral health professionals, mental health professionals, to ensure personal protective equipment for all of them; flexible work management, guidelines for vulnerable groups at greater risk of damage and lethality, mobile clinics and use of telemedicine.
\end{abstract}

Keywords: Primary health care. Unified Health System. Pandemics. Covid-19. Health services administration. Rapid review.

\footnotetext{
${ }^{1}$ Doutorado em Ciência da Informação na Universidade Federal do Rio de Janeiro e em Administração pela Fundação Getúlio Vargas.

${ }^{2}$ Doutoranda em Ciência da Informação PPGCI/IBICT/UFRJ; Mestre em Biblioteconomia PPGB/UNIRIO; Bibliotecária da Universidade Federal do Rio de Janeiro

3 Doutorado em Ciência da Informação pela Universidade de Brasília.

${ }^{4}$ Mestrado em Biblioteconomia pela Universidade Federal do Estado do Rio de Janeiro.
} 


\section{INTRODUÇÃO}

O objetivo desta revisão sistemática é identificar as possibilidades de cuidados básicos em nível primário que podem ser executados para enfrentar a pandemia da Covid-19. Assim, buscar-se-á na literatura sugestões de agenda das ações e serviços para o Sistema Único de Saúde (SUS). Na pandemia de Covid-19, notificada em 2020, cabe ao SUS garantir assistência à maioria da população brasileira, visto que o contexto pandêmico demanda ações emergenciais do sistema de saúde.

Após um ano de pandemia, com a aprendizagem que ela proporcionou, cabe avaliar o que foi feito em diferentes países e buscar os melhores caminhos para o Sistema Único de Saúde. Acredita-se que incluem uma agenda nacional de atenção básica de saúde, para enfrentar os desafios da pandemia, focada principalmente em ações e serviços de vigilância em saúde e de assistência básica.

A experiência com a pandemia da Covid-19 evidencia a necessidade e a efetividade da intervenção do Estado para garantir ações e serviços de saúde para todos. No caso brasileiro, reafirma-se a correção das diretrizes da Constituição Federal para a construção do SUS: ações e serviços coordenados, integrados, hierarquizados e descentralizados.

\section{METODOLOGIA}

A metodologia adotada nesta pesquisa é a revisão sistemática rápida, que consiste numa variante metodológica da revisão sistemática de literatura. As revisões rápidas em saúde são comumente usadas nas crises com doenças emergentes, formulação de políticas públicas, pesquisas de eficácia comparativa e avaliação de tecnologias. Seu processo é mais otimizado do que o de uma revisão sistemática, mas mantém os princípios metodológicos de síntese do conhecimento (TRICCO; LANGLOIS; STRAUS, 2017).

Geralmente, as revisões sistemáticas utilizam pesquisas primárias para que, com base em evidências científicas, seja possível ter um resumo atualizado do estado da arte das pesquisas sobre determinada temática (HIGGINS et al., 2019). Assim, revisões sistemáticas anteriores, artigos de opinião e editorais, justamente, por não atenderem o critério de serem pesquisas primárias não devem ser incluídas em uma nova revisão.

A revisão sistemática envolve a formulação da questão de pesquisa, encontrar os estudos, avaliar e selecionar, resumir e sintetizar o conteúdo relevante e determinar a aplicabilidade dos resultados (GLASZIOU et al., 2001). Neste sentido, para esta pesquisa foi 


\section{ARTIGO}

INOVAÇÃo

construída a seguinte questão: O que pode ser feito no nível da atenção primária em termos de cuidados básicos de saúde contra a pandemia de Covid-19?

Esta questão de pesquisa é constituída por três eixos conceituais que servem de apoio para a construção da estratégia de busca utilizada nas bases, sendo eles: atenção primária, Covid-19 e cuidados básicos de saúde. Esses conceitos foram traduzidos para termos em português, inglês e espanhol, que compuseram a expressão de busca geral.

As bases de dados utilizadas nesta pesquisa foram a PUBMED, a LILACS e a Scielo Saúde Pública. A PUBMED por ser uma base que tem grande poder de indexação de periódicos científicos, a LILACS por ter abrangência na América Latina e a Scielo Saúde Pública por ter uma abrangência nacional. Os termos utilizados na expressão de busca foram adaptados para cada base de acordo com o Medical Subject Headings (MeSH).

Na PUBMED, foi empregada a seguinte expressão de busca: ((Severe Acute Respiratory Syndrome Coronavirus 2[Supplementary Concept] OR Covid-19[Supplementary Concept] OR Severe Acute Respiratory Syndrome Coronavirus 2[tiab] OR Coronavirus Disease 2019[tiab] OR 2019 Novel Coronavirus[tiab] OR Wuhan Coronavirus[tiab] OR Covid-19[tiab] OR SARS-CoV-2[tiab] OR 2019-nCoV[tiab]) AND (Primary Health Care[mh] OR Primary Health Care[tiab] OR Primary Care[tiab] OR General Practitioners[mh] OR General Practitioner*[tiab] OR Family Health[mh] OR Family Health[tiab] OR Community Health Workers[mh] OR Community Health Worker*[tiab] OR Physicians, Family[mh] OR Family Physician*[tiab] OR Ambulatory Care[mh] OR Ambulatory Care[tiab] OR Ambulatory Treatment[tiab] OR “Out-of-Hospital Care"[tiab] OR "Out-of-Hospital Treatment"[tiab] OR "Active Search"[tiab]) AND (“Medical Care”[tiab]))

Além da construção da questão de pesquisa, dos critérios de inclusão e da definição das bases de dados é necessário que sejam estabelecidos os critérios de inclusão e exclusão dos estudos que serão ou não analisados.

Na presente revisão foram excluídos artigos focados em: revisão de literatura, opinião ou editorial; cuidados especializados; tecnologias complexas; idioma diferente do português, inglês ou espanhol; bem como, aqueles que não estavam acessíveis nas bases de dados disponíveis no Brasil. Os critérios de exclusão permitem, adicionalmente, a exclusão dos artigos que tragam resultados que não estão no escopo da pesquisa (PETTICREW; ROBERTS, 2006).

\section{RESULTADOS}

P2P \& INOVAÇÃO, Rio de Janeiro, v. 7 n. 2, p.140-163, Mar/Ago. 2021. 


\section{ARTIGO}

- INOVAÇÃo

Para esta pesquisa foram realizadas duas buscas em períodos diferentes. Em 14 de setembro de 2020 foram realizadas buscas nas bases de dados LILACS e PUBMED que resultaram na localização de 147 artigos. Em 15 de outubro de 2020 foi realizada a busca na base de dados Scielo Saúde Pública que resultaram em 30 artigos. Esses dados foram exportados para o programa de gestão de referências bibliográficas Zotero. Foram excluídos 46 itens duplicados. Os metadados das publicações foram exportados para o MS-Excel. A partir da leitura duplo cega dos resumos dos artigos foram aplicados os critérios de exclusão descritos na metodologia. Com a aplicação desses critérios foram excluídos do escopo da pesquisa 107 artigos. Desta forma, 24 artigos foram selecionados para a leitura do texto completo, análise de seu conteúdo e a sintetização dos resultados dos estudos primários. Essas etapas estão representadas na Figura 1. 
Figura 1 - Etapas do processo de recuperação de documentos para a RSL

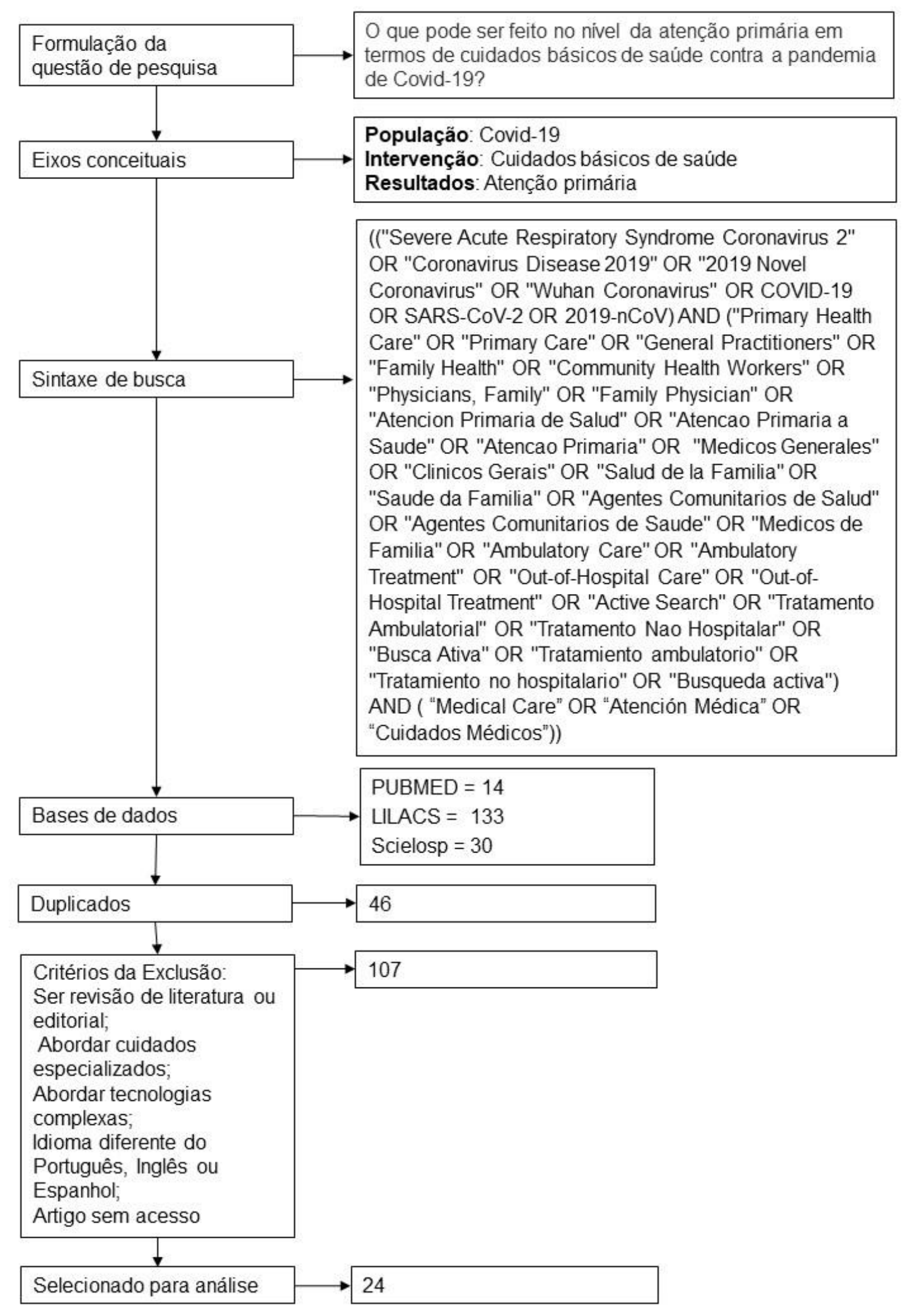

Fonte: Elaborado pelos autores (2020) 


\section{ARTIGO}

INOVAÇÃO

$\mathrm{Na}$ figura 1, é possível visualizar as etapas percorridas na pesquisa. Essas etapas resultaram na seleção de 24 artigos sintetizados no Quadro 1.

Quadro 1: Artigos selecionados para RSL

\begin{tabular}{|c|c|c|c|}
\hline Autor & Ano & Título & Foco \\
\hline CORBURN, J.; et al & 2020 & $\begin{array}{l}\text { Slum Health: Arresting Covid-19 and } \\
\text { Improving Well-Being in Urban } \\
\text { Informal Settlements }\end{array}$ & $\begin{array}{l}\text { Assentamento urbano } \\
\text { informal } \\
\text { (Estados Unidos) }\end{array}$ \\
\hline $\begin{array}{l}\text { BLAZEY-MARTIN, D.; } \\
\text { et al. }\end{array}$ & 2020 & $\begin{array}{l}\text { Primary Care Population Management } \\
\text { for Covid-19 Patients }\end{array}$ & $\begin{array}{l}\text { Cuidados primários } \\
\text { (Boston) }\end{array}$ \\
\hline $\begin{array}{l}\text { SWAZO, N.K.; } \\
\text { TALUKDER, M. M. H.; } \\
\text { AHSAN, M. K. }\end{array}$ & 2020 & $\begin{array}{l}\text { A Duty to treat? A Right to refrain? } \\
\text { Bangladeshi physicians in moral } \\
\text { dilemma during Covid-19 }\end{array}$ & Dilema moral (Bangladesh) \\
\hline DOOLITTLE, B. R.; et al. & 2020 & $\begin{array}{l}\text { The day the residents left: lessons } \\
\text { learnt from Covid-19 for ambulatory } \\
\text { clinics. }\end{array}$ & $\begin{array}{l}\text { Cuidados primários (New } \\
\text { Haven, Connecticut) }\end{array}$ \\
\hline $\begin{array}{l}\text { OLAGUNDOYE, O.; } \\
\text { ENEMA, O.; } \\
\text { ADEBOWALE, A. }\end{array}$ & 2020 & $\begin{array}{l}\text { Recommendations for a national } \\
\text { Coronavirus disease } 2019 \text { response } \\
\text { guideline for the care of older persons } \\
\text { in Nigeria during and post-pandemic: } \\
\text { A family physician's perspective. }\end{array}$ & $\begin{array}{l}\text { Cuidado dos idosos } \\
\text { (Nigéria) }\end{array}$ \\
\hline $\begin{array}{l}\text { MOTLHATLHEDI, K.; et } \\
\text { al. }\end{array}$ & 2020 & $\begin{array}{l}\text { Coronavirus disease } 2019 \text { in } \\
\text { Botswana: Contributions from family } \\
\text { physicians. }\end{array}$ & $\begin{array}{l}\text { Médicos de família } \\
\text { (Botswana) }\end{array}$ \\
\hline $\begin{array}{l}\text { MORREEL, S.; PHILIPS, } \\
\text { H.; VERHOEVEN, V. }\end{array}$ & 2020 & $\begin{array}{l}\text { Organisation and characteristics of } \\
\text { out-of-hours primary care during a } \\
\text { Covid-19 outbreak: A real-time } \\
\text { observational study. }\end{array}$ & $\begin{array}{l}\text { Consultas de atenção } \\
\text { primária fora do expediente } \\
\text { (Bélgica) }\end{array}$ \\
\hline JENKINS, L. S.; et al. & 2020 & $\begin{array}{l}\text { The evolving role of family physicians } \\
\text { during the coronavirus disease } 2019 \\
\text { crisis: An appreciative reflection. }\end{array}$ & Médico de família (Nigéria) \\
\hline OSENI, T. I. A.; et al. & 2020 & $\begin{array}{l}\text { The role of the family physician in the } \\
\text { fight against Coronavirus disease } 2019 \\
\text { in Nigeria. }\end{array}$ & Médico de família (Nigéria) \\
\hline SAMPA, M.B.; et al. & 2020 & $\begin{array}{l}\text { Redesigning Portable Health Clinic } \\
\text { Platform as a Remote Healthcare } \\
\text { System to Tackle Covid-19 Pandemic } \\
\text { Situation in Unreached Communities. }\end{array}$ & $\begin{array}{l}\text { Comunidades não } \\
\text { alcançadas com doenças não } \\
\text { transmissíveis (DNTs) } \\
\text { (Países em } \\
\text { desenvolvimento) }\end{array}$ \\
\hline YU, E.Y. T.; et al. & 2020 & $\begin{array}{l}\text { How are family doctors serving the } \\
\text { Hong Kong community during the } \\
\text { Covid-19 outbreak? A survey of } \\
\text { HKCFP members. }\end{array}$ & $\begin{array}{l}\text { Médicos de família (Hong } \\
\text { Kong) }\end{array}$ \\
\hline LI, G.; et al. & 2020 & $\begin{array}{l}\text { What patients "see" doctors in online } \\
\text { fever clinics during Covid-19 in } \\
\text { Wuhan? }\end{array}$ & $\begin{array}{l}\text { Clínicas de febre online } \\
\text { (Wuhan) }\end{array}$ \\
\hline
\end{tabular}




\section{ARTIGO}

INOVAÇÃo

\begin{tabular}{|c|c|c|c|}
\hline LIU, Y.; et al. & 2020 & $\begin{array}{l}\text { A Covid-19 Risk Assessment } \\
\text { Decision Support System for General } \\
\text { Practitioners: Design and } \\
\text { Development Study. }\end{array}$ & $\begin{array}{l}\text { Desenvolvimento de } \\
\text { dispositivo móvel para } \\
\text { Covid-19 para auxiliar no } \\
\text { diagnóstico dos médicos de } \\
\text { clínica geral (China) }\end{array}$ \\
\hline VERHOEVEN, V.; et al. & 2020 & $\begin{array}{l}\text { Impact of the Covid-19 pandemic on } \\
\text { the core functions of primary care: } \\
\text { will the cure be worse than the } \\
\text { disease? A qualitative interview study } \\
\text { in Flemish GPs. }\end{array}$ & Cuidados clínicos (Flandres) \\
\hline LUSIGNAN, S. de; et al. & 2020 & $\begin{array}{l}\text { The Oxford Royal College of General } \\
\text { Practitioners Clinical Informatics } \\
\text { Digital Hub: Protocol to Develop } \\
\text { Extended Covid-19 Surveillance and } \\
\text { Trial Platforms. }\end{array}$ & $\begin{array}{l}\text { Plataforma de teste e } \\
\text { vigilância estendida (Reino } \\
\text { Unido) }\end{array}$ \\
\hline SCHINKÖTHE, T.; et al. & 2020 & $\begin{array}{l}\text { A Web- and App-Based Connected } \\
\text { Care Solution for Covid-19 In- and } \\
\text { Outpatient Care: Qualitative Study } \\
\text { and Application Development. }\end{array}$ & $\begin{array}{l}\text { Aplicativo para auxílio na } \\
\text { triagem de pacientes } \\
\text { (Argentina, Alemanha, Irã, } \\
\text { Itália, Portugal, Suíça e } \\
\text { Estados Unidos) }\end{array}$ \\
\hline GOLDFIELD, N. I.; et al. & 2020 & $\begin{array}{l}\text { Covid-19 Crisis Creates Opportunities } \\
\text { for Community-Centered Population } \\
\text { Health: Community Health Workers at } \\
\text { the Center. }\end{array}$ & $\begin{array}{l}\text { Agente comunitário de } \\
\text { Saúde (Estados Unidos) }\end{array}$ \\
\hline VIVANTI, A. J.; et al. & 2020 & $\begin{array}{l}\text { Follow-up for pregnant women during } \\
\text { the Covid-19 pandemic: French } \\
\text { national authority for health } \\
\text { recommendations. }\end{array}$ & $\begin{array}{l}\text { Acompanhamento de } \\
\text { gestantes (França) }\end{array}$ \\
\hline LUSIGNAN, S. de; et al. & 2020 & $\begin{array}{l}\text { Emergence of a Novel Coronavirus } \\
\text { (Covid-19): Protocol for Extending } \\
\text { Surveillance Used by the Royal } \\
\text { College of General Practitioners } \\
\text { Research and Surveillance Centre and } \\
\text { Public Health England }\end{array}$ & $\begin{array}{l}\text { Protocolo de vigilância } \\
\text { virológica (Reino Unido) }\end{array}$ \\
\hline MOLONEY, K.; et al. & 2020 & $\begin{array}{l}\text { Experiences and Insights from the } \\
\text { Early US Covid-19 Epicenter: A } \\
\text { Rapid Assessment Procedure } \\
\text { Informed Clinical Ethnography Case } \\
\text { Series. }\end{array}$ & $\begin{array}{l}\text { Atenção primária e } \\
\text { secundária (Estados Unidos) }\end{array}$ \\
\hline $\begin{array}{l}\text { KOENIG, K.L.; BEŸ, } \\
\text { C.K.; McDONALD, E. C. }\end{array}$ & 2020 & $\begin{array}{l}\text { 2019-nCoV: The Identify-Isolate- } \\
\text { Inform (3I) Tool Applied to a Novel } \\
\text { Emerging Coronavirus. }\end{array}$ & $\begin{array}{l}\text { Gestão de pacientes } \\
\text { suspeitos (Estados Unidos) }\end{array}$ \\
\hline LUCENA, E. H. G.; et al & 2020 & $\begin{array}{l}\text { Acesso em saúde bucal na atenção } \\
\text { básica antes e após o início da } \\
\text { pandemia de COVID-19 no Brasil. }\end{array}$ & $\begin{array}{l}\text { Assistência em saúde bucal } \\
\text { no contexto nacional } \\
\text { (Brasil) }\end{array}$ \\
\hline MENESES, A. S. de & 2020 & $\begin{array}{l}\text { Gerenciamento emergencial de } \\
\text { recursos da atenção primária à saúde } \\
\text { no enfrentamento à pandemia da } \\
\text { covid-19 }\end{array}$ & $\begin{array}{l}\text { Gestão de unidades de saúde } \\
\text { (Brasil) }\end{array}$ \\
\hline $\begin{array}{l}\text { SALES, C. M. M.; } \\
\text { SILVA, A.I. da; MACIEL, } \\
\text { E. L. N. }\end{array}$ & 2020 & $\begin{array}{l}\text { Vigilância em saúde da COVID-19 no } \\
\text { Brasil: investigação de contatos pela } \\
\text { atenção primária em saúde como } \\
\text { estratégia de proteção comunitária. }\end{array}$ & $\begin{array}{l}\text { Vigilância territorial para a } \\
\text { busca de contatos de casos } \\
\text { de COVID-19 (Brasil) }\end{array}$ \\
\hline
\end{tabular}

Fonte: Elaborado pelos autores (2020) 
Nos próximos parágrafos, a síntese dos artigos selecionados foi organizada por continentes e países. Dois artigos são intercontinentais. Essa estratégia de apresentação foi escolhida por ser de amplo conhecimento que os sistemas de saúde são diferentes em cada país. Logo, observar detalhadamente as estratégias utilizadas e as políticas adotadas em cada país e região, permite delinear um panorama das ações que estão sendo praticadas internacionalmente, independentemente do modus operandi de cada sistema de saúde.

\section{Ásia}

Estudo desenvolvido em Wuhan extraiu dados de registros clínicos em um sistema de informação médica, produzidos entre 24 de janeiro a 18 de fevereiro de 2020 em um hospital terciário, que ofereceu um serviço de clínica de febre online. Considerando os registros de 64.487 pacientes que compareceram à clínica de febre online, os autores esclarecem que as clínicas de febre online podem efetivamente amenizar o pânico dos pacientes, além disso, os médicos podem orientar pacientes com suspeita de Covid-19 a se isolar e se proteger. Estas clínicas também podem ajudar a hierarquizar a atenção e a prevenir a infecção cruzada (LI; CHEN; DENG, 2020).

Estudo também desenvolvido na China apresentou um dispositivo móvel para Covid19 a fim de auxiliar os médicos de clínica geral na triagem e diagnóstico dos pacientes, permitindo antecipar a detecção, a notificação, o isolamento e o tratamento de Covid-19. O aplicativo usa inteligência artificial para o diagnóstico dos pacientes que respondem ao questionário, permitindo ao paciente a escolha em ir ou não as clínicas de atenção primária para exames complementares em caso de suspeita da Covid-19 (LIU et al, 2020).

Uma pesquisa transversal online tendo como participantes membros do Colégio de Médicos de Família de Hong Kong (HKCFP) avaliou se os médicos de família estavam preparados para lidar com a Covid-19. Dos 491 médicos respondentes, 393 tinham especialização em medicina de família, 236 trabalhavam em clínicas de atenção primária pública e 242 no setor privado. A maioria estava prestando serviços de atendimento à saúde, 310 deles sentiram que havia a necessidade de maiores esclarecimentos sobre a doença.

Os médicos de família em unidades de saúde públicas usaram as diretrizes do Center for Health Protection ou da Autoridade Hospitalar para tomada de decisão sobre a doença. Quase todos os entrevistados adotaram o uso de máscaras durante a consulta e lavagem das mãos antes do atendimento ao paciente.

As medidas sugeridas pelos médicos foram a necessidade de fornecimento de EPI de qualidade, uma política de saúde pública mais eficaz, controle das fronteiras com isolamento 
de pacientes vindos do exterior, criação de uma Autoridade de Atenção Primária para aumentar a eficiência e a eficácia da distribuição da atenção primária, disponibilidade de testes rápidos e ressaltaram a importância da educação em saúde para a prática de controle da doença.

As preocupações dos médicos estavam centradas em falta de EPIs, impossibilidade de prestar os serviços clínicos e na desinformação da população gerada pela propagação de notícias falsas. Os autores destacam o preparo dos médicos de família para atuarem em Hong Kong e a necessidade de as autoridades de saúde melhorarem a definição dos papéis desempenhados por eles, bem como a coordenação entre os serviços de atenção primária públicos e privados (YU et al., 2020).

Um estudo em Bangladesh reflete sobre o dilema moral enfrentado pelos médicos frente às adversidades enfrentadas para tratar de pacientes com Covid-19. Os autores trazem o conceito de dilema moral proposto pelo filósofo Michael Shaw Perry e as discussões éticas kantianas para refletirem sobre o enigma moral do atendimento médico a pacientes com Covid-19.

Em Bangladesh, alguns fatores evidenciaram a necessidade dessa reflexão, sendo eles: 1) a falta de equipamento de proteção individual (EPI) em hospitais e clínicas, colocando agentes de saúde e pacientes em risco de contágio; 2) leitos de UTI e enfermarias de isolamento em número limitado, associado a falta de equipamentos adequados para tratamento de pacientes positivos para Covid-19; 3) o volume de pacientes e tempo prolongado de internação associado a capacidade de resposta dos hospitais; 4) o estigma social causado pela Covid-19 impulsiona pacientes a não se declararem positivos, o que coloca em risco de contágio tanto os agentes de saúde como outros pacientes; 5) hospitais privados não tratam pacientes com Covid-19, sendo estes encaminhados para hospitais públicos. Associado a esses fatores está a falta de testes para identificar os pacientes positivos, o que gera incertezas no manejo desses pacientes.

Como resultado dessas reflexões os autores propõem diretrizes éticas para médicos e autoridades hospitalares, que incluem: 1) médicos e autoridades hospitalares devem reconhecer o dilema moral existente e respeitar a decisão individual do médico; 2) apesar do direito profissional do médico de não exercer suas atividades, ele deve exercê-la com base no conceito de emergência moral proposto por Kant; 3) uso de EPI e protocolos de controle de infecção; 4) entender o fato de médicos terem deveres familiares que se sobrepõe ao dever de tratar; 5) as autoridades administrativas do hospital devem reconhecer as incertezas da apresentação clínica aguda da Covid-19, respeitando as decisões de tratamento propostas 
pelos médicos; 6) incentivo a troca de informações entre médicos para que estes possam compartilhar experiências clínicas; 7) administradores hospitalares ou autoridades governamentais devem fornecer adequadamente os EPIs e os protocolos de atendimento a pacientes infectados; 8) na falta de EPI, o médico tem o direito de abster-se de tratar pacientes com Covid-19; 9) médicos que deixam de dar assistência devido à falta de EPI não devem sofrer sanções por parte das autoridades hospitalares e governamentais; 10) médicos de salas de emergência e ambulatórios devem fornecer um atendimento mínimo de cuidados médicos avaliativos e estabilizadores para pacientes com suspeita de infecção pela Covid-19 (SWAZO; TALUKDER; AHSAN, 2020).

\section{Europa}

Estudo no Reino Unido descreve o protocolo de desenvolvimento de duas plataformas pelo Centro Digital de Informática Clínica do Oxford Royal College of General Practitioners (ORCHID): a plataforma de ensaios clínicos e a plataforma de vigilância estendida. O objetivo dessas plataformas é dar suporte informacional para profissionais da atenção primária. Para tanto, foram aplicados os princípios de dados localizáveis, acessíveis, interoperáveis e, portanto, reutilizáveis no desenvolvimento das plataformas a fim de coletar os dados individuais dos pacientes com foco tanto na assistência como no desenvolvimento de pesquisas (LUSIGNAN et al., 2020a).

Também no Reino Unido, estudo relata o desenvolvimento de um protocolo de vigilância virológica da Covid-19 pelo Centro de Pesquisa e Vigilância (RSC) do Royal College of General Practitioners (RCGP) e a Public Health England (PHE) que possui uma rede de mais de 500 clínicas gerais na Inglaterra. O objetivo deste estudo é observar a população assintomática, casos ambulatoriais com infecções respiratórias, taxa e o padrão de disseminação da Covid-19 e avaliar a política de contenção.

Para tanto, os autores destacaram cinco estratégias com seus modos de ação: 1) registro de prontuário médico: foi criado um formulário com codificação agrupada ontologicamente em quatro categorias: "definido", "provável", "possível" e "não é um caso"; 2) extensão da vigilância virológica: criação do Observatório da Covid-19 para exposição dos dados coletados a partir da codificação criada no formulário; 3) coleta de amostra de sorologia por faixa etária: foram coletadas amostras semanais de pacientes que comparecerem nos consultórios para coleta de sangue de rotina; 4) Coleta de amostra de soro de convalescente: monitoramento de pacientes que foram positivo para Covid-19 com amostra virológica aguda; 
5) Curadoria de dados: incluindo o uso de princípios de dados localizáveis, acessíveis, interoperáveis e reutilizáveis (LUSIGNAN et al., 2020b).

Focando-se nos cuidados médicos na região de Flandres, Bélgica, um estudo investigou as consequências do surto da Covid-19 nas competências essenciais da clínica geral (GP).

A partir de entrevistas com 132 médicos, os autores buscaram entender sete aspectos com seus modos de ação: 1) gestão de atenção primária, observando o uso de telemedicina na triagem de pacientes; 2) cuidado centrado na pessoa, observando a preocupação dos médicos no uso de telemedicina via telefone, pois o telefone dificulta a comunicação não verbal gerada pela capacidade limitada de alguns pacientes em verbalizar suas necessidades, além das barreiras interculturais e de linguagem; 3) habilidades de resolução de problemas, observando que a prioridade de atenção à Covid-19 dificulta o tratamento de doenças crônicas; 4) atendimento integral, identificando a necessidade de divulgação de informações confiáveis para os pacientes por meio de diferentes mídias, sites e panfletos com informações sobre a Covid-19; 5) orientação à comunidade, verificando a importância de orientar os pacientes quanto ao distanciamento social e às vulnerabilidades; 6) visão holística, verificando a necessidade de cuidados psicossociais para evitar sintomas de solidão, depressão e violência intrafamiliar; 7) autoproteção e autocuidado, observando que os médicos estão sob uma alta carga psicológica devido ao medo de contrair a doença, de ser assintomático e contaminar pacientes e familiares, bem como devido a escassez de EPIs.

Os resultados do estudo deram destaque a alguns pontos. Ente eles: 1) os cuidados regulares das doenças crônicas a longo prazo serão prejudicados devido ao foco na triagem e atendimento aos casos de Covid-19; 2) a escassez de EPIs para cuidados de autoproteção preocupa os médicos no controle de contágio da doença; 3) as consequências psicológicas e socioeconômicas; 4) necessidade de uma melhor adaptabilidade das anotações das consultas por telefone; 5) preocupação com a educação médica, que vem sendo prejudicada pela falta de contato dos estagiários com os pacientes, sugerindo a capacitação dos alunos em telessaúde (VERHOEVEN et al, 2020).

Também na Bélgica, um estudo observacional descreveu a organização e as características das consultas na atenção primária durante cinco fins de semana no pico de um surto de Covid-19, comparando-o a um período semelhante em 2019. Como resultado, observou-se que os clínicos gerais podem reorganizar rapidamente o atendimento nos finais de semana para lidar com os fluxos de pacientes com suspeita de Covid-19. Quarenta por cento dos contatos realizados no final de semana na atenção primária foram atendidos apenas 
por meio de consultas telefônicas. Assim, recomenda-se viabilizar uma consulta médica por telefone a todos os pacientes e não depender da triagem realizada por auxiliares, que terão que diferenciar entre uma condição infecciosa e uma condição normal. Por outro lado, os autores ressaltam que a demanda por consultas presenciais diminuiu drasticamente, provocando questionamentos sobre a segurança do paciente com condições não relacionadas a Covid-19 (MORREEL; PHILIPS; VERHOEVEN, 2020).

Um estudo na França preocupado com o tratamento das gestantes durante a pandemia construiu método de resposta rápida para a Autoridade Nacional de Saúde da França (Haute Autorité de Santé; HAS), a fim de formular recomendações para garantir o atendimento de gestantes durante a pandemia da Covid-19. As recomendações propostas foram: 1) monitorar as mulheres grávidas durante a pandemia; 2) todas devem comparecer a 3 consultas com ultrassom; 3) consultas pessoais a partir do terceiro trimestre; 4) acompanhamento de mulheres de baixo risco obstétrico pessoalmente; 5) acompanhamento de mulheres com risco de complicações obstétricas devem ser adaptados; 6) apoio psicossocial; 7) admissão para parto e cuidados pós-parto; 8) apoio de familiares e parceiros; 9) triagem de mulheres grávidas na admissão; 10) adaptação do trabalho em equipe; 11) suporte para atendimento ambulatorial; 12) monitorar casos de mulheres grávidas com suspeita ou confirmação de Covid-19; 13) gestão de emergência obstétrica; 14) protocolo de gestão de hospitalização (VIVANTI et al, 2020).

\section{América}

Estudo aplicado em um grande centro médico acadêmico em Boston, nos Estados Unidos, relata as mudanças na gestão da população nas práticas da atenção primária a partir da projeção, implementação e avaliação de um algoritmo, um registro médico eletrônico (EMR) e um relatório populacional para o tratamento remoto de pacientes com Covid-19. O centro médico atende 40.000 pacientes, com equipe formada por 40 médicos assistentes, 76 residentes de medicina interna, cinco assistentes médicos (PAs), cinco enfermeiros (NPs) e uma equipe de enfermeiras registradas (RNs), todos trabalhando presencial e remotamente. Com o início da pandemia a gestão de atendimento ficou dividida em um dia de atendimento presencial e quatro dias de atendimento remoto. Em 27 de abril de 2020, 305 pacientes foram diagnosticados com Covid-19 a partir de teste de RT-qPCR. Destes 305 casos, 196 voltaram a linha de base, 54 foram admitidos em hospitais, seis morreram e 40 receberam alta (BLAZEY-MARTIN et al.,2020). 
Estudo realizado em New Haven, Estados Unicos, relata mudanças de procedimentos e incorporação da telessaúde na medida em que os médicos residentes tiveram que sair dos ambulatórios para compor unidades de terapia intensiva (UTIs) improvisadas e enfermarias exclusivas para Covid-19. Os 40.000 pacientes das clínicas ambulatoriais passaram a ser atendidos remotamente. Para isso, era necessário que todos os pacientes fossem inscritos no portal do paciente ou no prontuário eletrônico, pois desta forma seria possível atender com chamadas virtuais. Esse movimento de inscrição mobilizou estudantes de medicina voluntários e médicos assistentes para lançar uma campanha para aumentar o cadastro dos pacientes. Apesar dos esforços, os autores relataram dificuldades para o uso das plataformas de telessaúde, sendo uma parcela dos pacientes atendida por visitas ou por telefone (DOOLITTLE et al., 2020).

Estudo desenvolvido nos Estados Unidos relatou as modificações desenvolvidas na ferramenta 3I (Identificar, Isolar, Informar) desenvolvida para monitorar a doença do Ebola e que permitiriam o gerenciamento dos pacientes portadores da Covid-19. Desta forma, pacientes identificados positivamente para o vírus são isolados; e para proteção é utilizado a máscara N95 pelos agentes de saúde e uso de máscara cirúrgica pelos pacientes. O resultado do estudo aponta que a ferramenta Identify-Isolate-Inform serve como recurso para profissionais de saúde que precisam fazer avaliações claras e rápidas quando confrontado com pacientes potenciais (KOENING; BEY; MCDONALD, 2020).

Estudou-se as condições de moradia e a propagação da Covid-19 nos assentamentos informais do Sul Global. Os autores propõem oito estratégias voltadas a diminuir a disseminação da doença, melhorar a probabilidade de atendimento médico e melhorar e proteger econômica, social e fisicamente os moradores dessas localidades. As oito estratégias consistem em: 1) comitê emergencial de favelas, nessa ação seriam usados as ONGs conhecidas pela comunidade; 2) criação de moradia regular, procurar locais próximos aos assentamentos para criar casas regulares, preservando assim o vínculo social; 3) renda emergencial, providenciar pagamentos emergenciais devido à perda salarial durante a pandemia; 4) agentes comunitários, incorporar mais agentes de saúde comunitários nessas localidades; 5) saneamento básico, providenciar redes de água e esgoto; 6) assistência alimentar, governo deve apoiar entidades religiosas e assistenciais para que os alimentos sejam cozidos para todos, garantindo nutrição adequada; 7) coleta de resíduos sólidos, estimular reciclagem e coleta de resíduos sólidos; 8) plano de emergência, implantação de clínicas móveis para monitoramento da doença, incluindo ambulância e bombeiros. Os autores destacam a necessidade de pesquisas multidisciplinares para identificar as estratégias 
utilizadas pela população para enfrentar a Covid-19, além de compreender os impactos envolvidos nesse processo (CORBURN et al, 2020).

Um estudo realizado nos Estados Unidos estabeleceu diretrizes para implantação do cuidado comunitário de saúde de base populacional com base na experiência do Estado de Massachusetts. Os autores destacam o papel dos agentes comunitários de saúde nesse processo. A proposta consiste em: 1) acompanhamento epidemiológico das estratégias de saúde e da aceitação social; 2) orientação a comunidade com informações confiáveis sobre a doença: sintomas, formas de proteção; 3) controle e isolamento de pacientes infectados; 4) vigilância ativa de novos casos; 5) mitigação da doença com medidas de distanciamento social, fechamento de escolas, telemedicina; 6) testes sorológicos para estimar infecções atuais e anteriores; 7) preparação do sistema de saúde; 8) pesquisa continuada para entender a evolução da doença com base em evidências científicas. Os autores destacam o papel essencial dos agentes comunitários de saúde, pois este são por definição membros de confiança da comunidade, além de possuir um enorme conhecimento da comunidade atendida, permitindo que sejam elo entre os serviços sociais, os serviços de saúde e a comunidade (GOLDFIELD et al., 2020).

Outro estudo desenvolvido nos Estados Unidos utilizou a Etnografia Clínica Informada do Procedimento de Avaliação Rápida (RAPICE) para desenvolver casos clínicos e observação participante para avaliar intervenções centradas no paciente e integradas por pares após lesão traumática. As estratégias sugeridas foram: 1) redirecionar os pacientes para locais com menor incidência de risco de contaminação por Covid-19; 2) avaliar risco de exposição a Covid-19 por pacientes com condições crônicas; 3) adoção de intervenções para estratégias de prevenção a Covid-19; 4) distanciamento social para cuidados médicos quando possível; 5) cuidados com os cuidadores da linha de frente para evitar contágio da Covid-19 (MOLONEY et al., 2020).

Estudo realizado no Brasil, a partir do Sistema de Informação em Saúde da Atenção Básica (SISAB), coletou coletados dados sobre o número de atendimentos por abscesso dentário e dor de dente, comparando o primeiro trimestre (janeiro a abril) de 2019 e de 2020. Observou-se que o acesso à saúde bucal na atenção básica foi reduzido em 2020, fenômeno que, provavelmente, irá impactar negativamente os dados epidemiológicos de saúde bucal no Brasil (LUCENA et al., 2020).

Estudo realizado no Brasil, considerando o contexto de 30 unidades básicas de saúde, contendo 170 equipes de saúde da família com 604.772 pessoas adscritas, constata que a pandemia exige uma agilidade e flexibilidade decisória. Destaca: 1) o êxito em manter a força 
de trabalho saudável e operante, além de dar apoio aos hospitais de referência; 2) a necessidade de prorrogação de receitas de uso contínuo e dispensação rápida nas farmácias; 3 ) o uso de tendas, drive thru, pátios de escolas para o sucesso da campanha de vacina; 4) flexibilizar as entregas sem prejuízo no financiamento dos serviços, que tiveram suas metas suprimidas em função do isolamento social; 5) aumentar o rigor e a frequência de limpeza nas unidades de saúde; 6) estabelecer estratégia de comunicação em massa por aplicativos e por canais de comunicação com lideranças comunitárias do território; 7) promover mobilizações e participação da comunidade em campanhas de conscientização sobre a pandemia por meio de estratégias de comunicação popular variadas (panelaço, apitaço, etc) (MENESES, 2020).

A racionalização dos recursos e a estruturação de fluxos de atenção, que direcionem o acompanhamento dos casos com responsabilidade territorial e priorização do cuidado e do acompanhamento contínuo é uma preocupação presente em outro estudo realizado no Brasil, onde propõe-se um fluxograma para auxiliar de maneira estratégica a organização da rede de serviços da atenção primária em saúde, de base territorial (SALES; SilLVA; MACIEL, 2020).

\section{África}

Em Botswana, observou-se que o distanciamento social aumentou a necessidade do uso de plataformas comunicacionais online. Os médicos de família e os clínicos gerais utilizavam WhatsApp na troca de informação entre pares antes da pandemia. Com o surto houve a ampliação do uso de webinars e das mídias sociais para atendimento aos pacientes. Os autores destacam a importância das tecnologias da informação na atenção primária, ressaltando que os médicos particulares usam a telessaúde para atender seus pacientes, realidade que não é observada na saúde pública em decorrência de limitada conectividade e acesso aos prontuários dos pacientes. Os autores destacam que, com o isolamento social, os membros das comunidades ficaram mais vulneráveis e ressaltaram a importância do papel dos médicos de família nos cuidados a esses grupos. Apontaram que os médicos de família vêm realizando parcerias com organizações e empresas locais, além das igrejas. Alguns vem realizando atividades de ensino sobre Covid-19 nas comunidades, com foco na prevenção e diminuição de risco, e muitos são membros ativos das comunidades. Ressalta-se que os médicos de família dispensam cuidados contínuos a sua comunidade, relacionados à Covid-19 e às doenças crônicas (MOTLHATLHEDI et al., 2020).

Estudo na Nigéria apresenta as medidas estabelecidas pela Sociedade de Médicos da Família para o enfrentamento da Covid-19, tais como: 1) triagem, realizada por meio de enfermeiras e histórico clínico do paciente; 2) educação e aconselhamento, a partir de 
disseminação de informações aos pacientes por meio de folhetos impressos e por meio eletrônico; 3) áreas de espera sem aglomeração, implantar o distanciamento nos locais de espera e o uso de EPIs; 4) atenção comportamental à família, aconselhamento e atenção familiar para evitar violência doméstica, depressão e outros sintomas comportamentais; 5) cuidados domiciliares, utilizar o recurso de atendimento domiciliar para evitar superlotação em hospitais; 6) telemedicina, atendimento por telemedicina como forma de triagem para hospitais; 7) hospice e cuidados paliativos, cuidados de idosos e doentes terminais a partir da gestão de doenças, treinar os cuidadores para melhor atender aos idosos, além de usar o recurso de telemedicina para atendimento. Os resultados apontados pelos autores foram redução da sobrecarga nos hospitais e identificação de pacientes com Covid-19, permitindo diagnóstico e tratamento precoce (OSENI et al., 2020).

Um estudo na Nigéria propôs a construção de diretrizes voltadas ao cuidado de idosos, composta por 7 estratégias e seus modos de ação, quais sejam: 1) manter o idoso com condições crônicas longe das instalações de saúde, fazendo-se uso de telemedicina; 2) testes para Covid-19 priorizando os idosos, sobretudo, antes de serem internados; 3) distribuição de pacotes paliativos; envolvendo no processo de cuidado ao idoso as associações e ONGs, visando prover alimentos, máscaras e outros recursos que se fizerem necessários; 4) cuidar da saúde mental do idoso, disponibilizando apoio profissional de saúde mental aos idosos; 5) conscientizar a família e a comunidade a cuidar dos idosos (alimento, remédio, amparo emocional), envolvendo a família e a comunidade em uma rede colaborativa para compras de alimentos e medicamentos, evitando contato físico, mas dando suporte informacional e emocional aos idosos; 6) inclusão dos idosos nas diretrizes governamentais de cuidados à população idosa do Centro de Controle de Doenças da Nigéria (NCDC); 7) inclusão de cuidados domiciliares nos seguros saúde, visto que não existe uma política de saúde nacional para cuidados domiciliares (OLAGUNDOYE; ENEMA; ADEBOWALE, 2020).

Um estudo traz a reflexão de dez médicos de família e atendentes de medicina de família em sua primeira reunião trimestral do complexo de treinamento semirural da África do Sul. Os autores ressaltam a importância dos médicos de família e seus papéis centrados na comunidade, trabalhando em equipes multiprofissionais, com foco em saúde preventiva. Os desafios dos médicos de família durante a pandemia da Covid-19 estão no distanciamento social, na redução dos procedimentos eletivos e na minimização do contato médico-paciente.

As competências nas diversas disciplinas clínicas, de liderança e comunicação, de colaboração dentro das equipes e a integração dos médicos de família com as comunidades 
são as características necessárias para que se possa responder de forma adequada a essa crise sanitária (JENKINS et al., 2020).

\section{Intercontinental}

Um estudo realizou entrevistas não estruturadas com médicos na Argentina, Alemanha, Irã, Itália, Portugal, Suíça e Estados Unidos a fim de identificar os desafios enfrentados no atendimento a pacientes com Covid-19. A partir das respostas obtidas foi desenvolvido o Covid-19 Caregiver Cockpit (C19CC), ferramenta gratuita, baseada na web e em aplicativos para auxiliar na avaliação dos pacientes. Os resultados alcançados com a ferramenta foram: 1) triagem do paciente e preparação da visita: os pacientes cadastrados no sistema passam a ter um relatório geral do quadro; 2) monitoramento remoto: pacientes podem continuar sendo observados após a alta; 3) uso em uma enfermaria de hospital: médicos recebem notificação de piora do paciente hospitalizado (SCHINKOTHE et al., 2020).

Estudo sobre comunidades não alcançadas com doenças não transmissíveis (DNTs) direcionadas a países em desenvolvimento aborda o uso de Remote Healthcare Systems (RHS) para minimizar o risco de contaminação pela Covid-19. Os autores usaram as diretrizes da Organização Mundial de Saúde e a estrutura de Design Science Research (DSR) para redesenhar a Portable Health Clinic (PHC) um serviço de saúde remoto. Com o uso do algoritmo de triagem (C-Logic) o paciente é classificado e encaminhado para o devido tratamento. Destacam que os serviços de Clínica de Saúde Portátil (APS), um tipo de RHS tem papel importante nas medidas de prevenção e fornecimento de informações aos pacientes.

O APS foi projetado para atender pacientes em comunidades não alcançadas de áreas remotas e foi testado em pacientes com DNTs. Para os autores o PHC pode contribuir: na triagem dos pacientes no nível primário; na redução da mortalidade e morbidades associadas em populações carentes em regiões remotas de países em desenvolvimento; na redução da carga da doença em pacientes DNTs, auxilia no processo de distanciamento ajudando no controle da transmissão; como alternativa de e-Saúde para serviços de saúde primária, educação preventiva em saúde e conscientização e estímulo ao bem estar; no controle de DNTs e diminuição da carga de doenças em comunidades-alvo; como forma de responder a emergências de saúde pública em grande escala em populações em risco (SAMPA et al., 2020).

\section{DISCUSSÃO}

P2P \& INOVAÇÃO, Rio de Janeiro, v. 7 n. 2, p.140-163, Mar/Ago. 2021. 
A organização das ações integradas em saúde parte da noção de que existem níveis de aplicação das medidas de controle e prevenção de agravos e danos à saúde. Leavell e Clark (1976) estabelecem estes níveis a partir da relação com evolução da história natural da doença. Os níveis primário, secundário e terciário se referem também ao nível de complexidade das ações. O Sistema Único de Saúde no Brasil trabalha com a ideia de ações integradas, que inclui a assistência à saúde, sem prejuízo das medidas preventivas.

O conceito de atenção primária de saúde que se usa neste artigo inclui, como diziam Leavell e Clark $^{28}$, a promoção da saúde e a sua proteção específica. Contudo, trabalha-se com o modo como se dá a operacionalização da atenção primária no SUS; equipes de saúde da família e agentes comunitários. O modo de operacionalizar do SUS tem duas fontes: o General Practitioner inglês e o médico de família cubano.

Trata-se de conceito adaptado de atenção primária no Brasil, que pode ser designado de atenção básica. Ele inclui promoção e proteção específica da saúde, especialmente por agentes comunitários. Entre as ações neste nível estão educação em saúde, vigilância epidemiológica e vacinação. A atenção básica contempla também os cuidados básicos da saúde, particularmente a assistência médica. Os atores privilegiados da atenção básica no Brasil são as equipes de saúde da família.

Considerando o esclarecimento acima e a questão inicial desta revisão de literatura: “ $o$ que pode ser feito em termos de cuidados básicos de saúde (ou de cuidados em nível de atenção primária) contra a pandemia de Covid-19?”, observa-se que, em diferentes países e continentes, algumas ações e tecnologias são frequentemente mencionadas para que se atue no nível de atenção primária de forma mais eficaz e sistematizada durante a pandemia. Nesse sentido, os estudos selecionados permitem esboçar uma agenda de atenção básica em saúde composta por 13 itens:

1. Estabelecer assistência de moradia e alimentar, em lugares e situações onde a população tenha vínculo estabelecido, sempre que possível envolvendo outros atores da sociedade, como famílias, organizações não governamentais e entidades religiosas conhecidas pela população;

2. Prover saneamento básico, particularmente água encanada e coleta de resíduos e de lixo;

3. Executar estratégias de disseminação de informações sobre a pandemia do Covid-19 e outras condições crônicas para a população, por meio de diferentes tecnologias e suportes informacionais, monitorando a aceitabilidade das informações; 
4. Criar plataformas para coleta de dados, testes e ensaios clínicos integradas, organizadas e sistematizadas de forma que se construa uma inteligência para a vigilância epidemiológica e para a assistência à saúde; Idealmente tais plataformas devem coletar informação e dados clínicos que possam também gerar conhecimento novo e evidências para melhores ações durante a pandemia;

5. Garantir equipamentos de proteção individual (EPI) e material para higienização das mãos para a equipe de saúde e para pacientes, de forma a gerar segurança física e psicológica durante a assistência à saúde;

6. Mobilizar agentes comunitários para executar estratégias e ações de saúde, incluindo a vigilância epidemiológica;

7. Garantir médicos de família, pois a sua ação centrada na comunidade e seu trabalho com equipes multiprofissionais permitem a concretização de uma atenção básica à saúde;

8. Oferecer assistência à saúde bucal em nível primária;

9. Oferecer assistência à saúde mental aos pacientes com Covid-19 e de seus familiares, a fim de diminuir o potencial estigma, o stress e a depressão gerados pela pandemia de Covid-19; Oferecer assistência à saúde mental dos trabalhadores das organizações de saúde;

10. Fazer a gestão das organizações de saúde ágil, flexível e discursiva;

11. Estabelecer diretrizes para assistência a grupos de maior risco e as populações vulneráveis, como pacientes idosos, mulheres grávidas, pacientes crônicos, pacientes em situação econômica crítica; sempre que possível envolvendo outros atores da sociedade, como famílias, ONGs e entidades religiosas; garantir a assistência farmacêutica a pacientes crônicos e idosos;

12. Organizar clínicas móveis, para que os profissionais de saúde possam ficar mais próximos da população;

13. Usar telemedicina, outros modos de assistência online e outras plataformas comunicacionais, para triagem e assistência aos pacientes com suspeita de Covid-19, com Covid-19 e com outras condições crônicas, incluindo-se aqui plataformas próprias de telemedicina ou, quando na impossibilidade dessas, recursos tecnológicos mais econômicos que possam ser usados tanto por profissionais da saúde quanto pela população para a assistência à saúde.

Os treze pontos da agenda de atenção básica estão sendo apresentados em nível de complexidade crescente, incluindo atividades de promoção da saúde, de vigilância epidemiológica e cuidados médicos simples. Cabe observar que a Covid-19 é uma doença emergente que ainda não tem medicação específica eficaz.

É importante destacar que os quatro primeiros itens desta agenda são atividades típicas da atenção primária de saúde, com ações para promoção e proteção específica da saúde: moradia 
com saneamento, água encanada e comida, além de educação em saúde, com disseminação de informações pertinentes e de vigilância epidemiológica de uma doença que emerge como pandemia global em 2020.

Outros 5 pontos referem-se a mobilizar agentes comunitários, médicos de família, profissionais de saúde bucal e profissionais de saúde mental, bem como garantir equipamentos de proteção individual para todos eles. Esta força de trabalho especial e protegida é fundamental para garantir a atenção básica, da vigilância epidemiológica à assistência básica em saúde mental, incluindo atividades de educação em saúde, assistência farmacêutica e de promoção de bem estar social.

Quatro itens da agenda estão direcionados para a organização da força de trabalho da atenção básica: gestão das organizações de saúde ágil, flexível e discursivo, diretrizes para grupos de vulneráveis e de maior risco de dano e letalidade, clínicas móveis e uso da telemedicina. Os grupos de maior risco incluem os idosos e os portadores de patologias crônicas. A demarcação territorial e populacional da atenção básica é fundamental para organização e gestão dos cuidados. A pandemia criou inúmeras possibilidades de uso de tecnologias digitais, do rastreamento de células a realização de teleconsultas.

\section{CONSIDERAÇÕES FINAIS}

No primeiro ano do enfrentamento da pandemia a maior parte dos países combinou medidas de isolamento social para reduzir a circulação do vírus com tratamento intensivo dos casos graves. No Brasil não houve a mobilização coordenada pelo Ministério da Saúde no nível primário, dos agentes comunitários e das equipes de saúde da família. As iniciativas neste sentido foram quase sempre das esferas municipais de governo, desarticuladas e isoladas.

O fato é que há certa exaustão da sociedade em manter medidas de isolamento social, bem como a experiência ensinou que os cuidados clínicos dos doentes devem ser realizados mais precocemente. É neste contexto que cabe e se torna pertinente o presente resgate de artigos sobre atenção básica contra a pandemia de Covid-19.

Assim, entendemos que se trata de observar a noção de hierarquização da rede de prevenção e de cuidados da saúde do Sistema Único de Saúde, tal como está disposto em sua lei orgânica. É hora de mobilizar equipes e agentes para executar medidas de atenção básica. A agenda apresentada na discussão pode servir para apoiar a definição de princípios e diretrizes das ações. 


\section{ARTIGO}

INOVAÇÃO

Cabe destacar medidas de atenção básica derivadas da referida agenda: seguro social para garantir condições de executar medidas de prevenção por toda a sociedade; educação em saúde, particularmente distanciamento social e procedimentos de higiene; vigilância epidemiológica, com notificação de casos e óbitos; testes para detecção de casos da doença, para orientar bloqueios; diagnóstico e tratamento precoce. É importante registrar as possibilidades de uso das tecnologias digitais de informação e comunicação em todas estas ações.

Outra questão relevante refere-se a execução de programa emergencial de vacinação em massa contra a Covid-19. O desenvolvimento de vacinas eficazes e seguras torna possível e necessário. As equipes de saúde da família e os agentes comunitários do SUS devem estar na linha de frente desta ação. Registre-se que o SUS tem um histórico de excelência na execução de programa nacional de imunizações.

Espera-se que a síntese desta revisão rápida apresentada contribua para a construção de uma agenda de atenção básica para enfrentamento da pandemia de Covid-19. A presente revisão foi elaborada entre setembro e outubro de 2020, após uma avalanche de estudos e pesquisas sobre a pandemia. Contudo, é importante observar que, em termos proporcionais, é pequena quantidade de trabalhos que se referem a atenção primária de saúde. Observados os critérios de seleção e de exclusão, restaram apenas 24 artigos para esta revisão.

Certamente, nos próximos meses e ano serão realizados mais estudos e pesquisas sobre atenção básica durante a pandemia, para enfrentá-la. Assim, esta revisão sistemática poderá ser atualizada por esta equipe ou por outras. Antes e acima de tudo, espera-se que aconteça a mobilização eficaz e efetiva de equipes de saúde da família do SUS, com os seus agentes comunitários. Assim como, espera-se que aconteça o controle da pandemia, com redução de agravos e danos. 


\section{REFERÊNCIAS}

BLAZEY-MARTIN, D.; et al. Primary Care Population Management for Covid-19 Patients. J Gen Intern Med., v. 35, n. 10, p. 3077-3080, out. 2020. Doi: 10.1007/s11606-020-05981-1. Epub. 27 jul. 2020. Disponível em https://link.springer.com/article/10.1007\%2Fs11606-02005981-1. Acesso em: 17 set. 2020

CORBURN, J.; et al. Slum Health: Arresting Covid-19 and Improving Well-Being in Urban Informal Settlements. J Urban Health., v. 97, n.3, p. 348-357, jun. 2020 Doi: 10.1007/s11524-020-00438-6. Disponível em: https://link.springer.com/article/10.1007/s11524-020-00438-6. Acesso em: 17 set. 2020.

DOOLITTLE, B. R.; et al. The day the residents left: lessons learnt from Covid-19 for ambulatory clinics. Fam Med Community Health., v.8, n.3, p. e000513, jul. 2020. Doi: 10.1136/fmch-2020-000513. Disponível em: https://fmch.bmj.com/content/8/3/e000513.long. Acesso em: 17 set. 2020

GLASZIOU, P.; et al. Systematic reviews in healthcare; a practical guide. Cambridge, UK, Cambridge University Press, 2001.

GOLDFIELD, N. I.; et al. Covid-19 Crisis Creates Opportunities for Community-Centered Population Health: Community Health Workers at the Center. J Ambul Care Manage., v. 43, n.3, p. 184-190, jul/set. 2020. Doi: 10.1097/JAC.0000000000000337. Disponível em: https://journals.lww.com/ambulatorycaremanagement/Fulltext/2020/07000/COVID_19_Crisis _Creates_Opportunities_for.2.aspx. Acesso em: 17 set. 2020.

HIGGINS, J. P. T.; et al. (ed.). Cochrane Handbook for Systematic Reviews of Interventions. 2. Ed. Chichester (UK): John Wiley \& Sons, 2019.

JENKINS, L. S.; et al.The evolving role of family physicians during the coronavirus disease 2019 crisis: An appreciative reflection. Afr J Prim Health Care Fam Med., v. 12, n. 1, p. e1-e4, jun. 2020. Doi: 10.4102/phcfm.v12i1.2478. Disponível em: https://www.ncbi.nlm.nih.gov/pmc/articles/PMC7343922/. Acesso em: 17 set. 2020

KOENIG, K.L.; BEŸ, C.K.; McDONALD, E. C. 2019-nCoV: The Identify-Isolate-Inform (3I) Tool Applied to a Novel Emerging Coronavirus. West J Emerg Med., v. 21, n. 2, p. 184190, mar. 2020. Doi: 10.5811/westjem.2020.1.46760. Epub 31 jan. 2020. Disponível em: https://www.ncbi.nlm.nih.gov/pmc/articles/PMC7081861/. Acesso em: 17 set. 2020.

LEAVELL, H.; CLARK, E. G. Medicina Preventiva. São Paulo: McGraw-Hill, 1976

LI, G.; et al. What patients "see" doctors in online fever clinics during Covid-19 in Wuhan? J Am Med Inform Assoc., v. 27, n.7, p. 1067-1071, jul. 2020. Doi: 10.1093/jamia/ocaa062. Disponível em: https://academic.oup.com/jamia/article/27/7/1067/5855846. Acesso em: 17 set. 2020

LIU, Y.; et al. Covid-19 Risk Assessment Decision Support System for General Practitioners: Design and Development Study. J Med Internet Res., v. 22, n. 6, p. e19786, jun. 2020. Doi: 10.2196/19786. Disponível em: https://www.jmir.org/2020/6/e19786/ Acesso em: 17 set. 2020 
LUCENA, E. H. G.; et al. Acesso em saúde bucal na atenção básica antes e após o início da pandemia de COVID-19 no Brasil. Pesquis Bras Odontopediatria Clin Integr. v. 20, p. 1-6, 2020. Doi 10.1590/SciELOPreprints.819. Epub 19 jun. 2020. Disponível em: https://preprints.scielo.org/index.php/scielo/preprint/view/819. Acesso em: 17 out. 2020

LUSIGNAN, S. de; et al. The Oxford Royal College of General Practitioners Clinical Informatics Digital Hub: Protocol to Develop Extended Covid-19 Surveillance and Trial Platforms.JMIR Public Health Surveill, v. 6, n, 3, p. e19773, jul/set. 2020a. Doi: 10.2196/19773. Disponível em: https://publichealth.jmir.org/2020/3/e19773/. Acesso em: 17 set. 2020.

LUSIGNAN, S. de; et al. Emergence of a Novel Coronavirus (Covid-19): Protocol for Extending Surveillance Used by the Royal College of General Practitioners Research and Surveillance Centre and Public Health England. JMIR Public Health Surveill, v. 6, n.2, p. e18606, abr./jun. 2020b. Doi: 10.2196/18606. Disponível em: https://publichealth.jmir.org/2020/2/e18606/. Acesso em: 17 set. 2020.

MENESES, A. S. de. Gerenciamento emergencial de recursos da atenção primária à saúde no enfrentamento à pandemia da covid-19. Scielo Prepr. 2020. Doi 10.1590/SciELOPreprints.557. Epub 29 mai 2020. Disponível em: https://preprints.scielo.org/index.php/scielo/preprint/view/557. Acesso em: 17 out. 2020

MOLONEY, K.; et al. Experiences and Insights from the Early US Covid-19 Epicenter: A Rapid Assessment Procedure Informed Clinical Ethnography Case Series. Psychiatry., v. 83, n. 2, p. 115-127, Summer 2020. Doi: 10.1080/00332747.2020.1750214. Epub 27 abr. 2020. Disponível em: https://www.tandfonline.com/doi/full/10.1080/00332747.2020.1750214. Acesso em: 17 set. 2020.

MORREEL, S.; PHILIPS, H.; VERHOEVEN, V. Organisation and characteristics of out-ofhours primary care during a Covid-19 outbreak: A real-time observational study. PLoS One., v. 15, n.8, p. e0237629, ago. 2020. Doi: 10.1371/journal.pone.0237629. Disponível em: https://journals.plos.org/plosone/article?id=10.1371/journal.pone.0237629 Acesso em: 17 set. 2020.

MOTLHATLHEDI, K.; et al. Coronavirus disease 2019 in Botswana: Contributions from family physicians. Afr J Prim Health Care Fam Med., v. 12, n.1, p. e1-e3, jul. 2020. Doi: 10.4102/phcfm.v12i1.2497. Disponível em: https://www.ncbi.nlm.nih.gov/pmc/articles/PMC7433220/. Acesso em: 17 set. 2020

OLAGUNDOYE, O.; ENEMA, O.; ADEBOWALE, A. Recommendations for a national Coronavirus disease 2019 response guideline for the care of older persons in Nigeria during and post-pandemic: A family physician's perspective. Afr J Prim Health Care Fam Med., v. 12, n. 1, p. e1-e3, ago. doi: 10.4102/phcfm.v12i1.2512. Disponível em: https://www.ncbi.nlm.nih.gov/pmc/articles/PMC7479416/. Acesso em 17 set. 2020.

OSENI, T.I.A.; et al. The role of the family physician in the fight against Coronavirus disease 2019 in Nigeria. Afr J Prim Health Care Fam Med., v. 12, n. 1, p.e1-e3, jun. 2020. Doi: 10.4102/phcfm.v12i1.2492. Disponível em: https://www.ncbi.nlm.nih.gov/pmc/articles/PMC7343949/. Acesso em: 17 set. 2020 
PETTICREW, Mark ; ROBERTS, Helen. Systematic reviews in the social sciences: a practical guide. Estados Unidos: Blackwell Publishing, 2006.

SALES, C. M. M.; SILVA, A.I. da; MACIEL, E. L. N. Vigilância em saúde da COVID-19 no Brasil: investigação de contatos pela atenção primária em saúde como estratégia de proteção comunitária. Epidemiol. Serv. Saúde, v. 29, n. 4, p. 2020373 set. 2020. Epub 23 Jul. 2020.Doi: 10.5123/s1679-49742020000400011. Disponível em: https://www.scielosp.org/article/ress/2020.v29n4/2020373/. Acesso em: 15 out. 2020

SAMPA, M.B.; et al. Redesigning Portable Health Clinic Platform as a Remote Healthcare System to Tackle Covid-19 Pandemic Situation in Unreached Communities. Int. J. Environ. Res. Public Health, v. 17, n. 13 p. 4709, jun. 2020. Doi: 10.3390/ijerph17134709 Disponível em: https://www.mdpi.com/1660-4601/17/13/4709. Acesso em: 17 set. 2020

SCHINKÖTHE, T.; et al. Web- and App-Based Connected Care Solution for Covid-19 Inand Outpatient Care: Qualitative Study and Application Development. JMIR Public Health Surveill, v. 6, n. 2, p. e19033, abr.-jun. 2020. Doi: 10.2196/19033. Disponível em: https://publichealth.jmir.org/2020/2/e19033/. Acesso em 17 set. 2020.

SWAZO, N. K.; TALUKDER, M. M. H.; AHSAN, M. K. A Duty to treat? A Right to refrain? Bangladeshi physicians in moral dilemma during Covid-19. Philos Ethics Humanit Med., v. 15, n. 1, p. 7, set. 2020. doi: 10.1186/s13010-020-00091-6. Disponível em: https://peh-med.biomedcentral.com/articles/10.1186/s13010-020-00091-6. Acesso em: 17 set. 2020

TRICCO, A. C.; LANGLOIS, E. V.; STRAUS, S. E. (ed.). Rapid reviews to strengthen health policy and systems: a practical guide. Geneva: World Health Organization, 2017. Disponível em: https://apps.who.int/iris/handle/10665/258698. Acesso em: 14 abr. 2020.

VERHOEVEN, V.; et al. Impact of the Covid-19 pandemic on the core functions of primary care: will the cure be worse than the disease? A qualitative interview study in Flemish GPs. BMJ Open, v. 10, n.6, p. e039674, jun. 2020. Doi: 10.1136/bmjopen-2020-039674.

Disponível em: https://bmjopen.bmj.com/content/10/6/e039674.long. Acesso em: 17 set. 2020

VIVANTI, A. J.; et al. Follow-up for pregnant women during the Covid-19 pandemic: French national authority for health recommendations. J Gynecol Obstet Hum Reprod., v. 49, n. 7, p. 101804, set. 2020. Doi: 10.1016/j.jogoh.2020.101804. Epub 11 mai. 2020. Disponível em: https://www.sciencedirect.com/science/article/abs/pii/S2468784720301471?via\%3Dihub. Acesso em: 17 set. 2020

YU, E.Y. T.; et al. How are family doctors serving the Hong Kong community during the Covid-19 outbreak? A survey of HKCFP members. Hong Kong Med J., v. 26, n. 3, p. 176183, jun. 2020. Doi: 10.12809/hkmj208606. Epub 1 jun. 2020 Disponível em: https://www.hkmj.org/abstracts/v26n3/176.htm. Acesso em: 17 set. 2020. 